\title{
NDVI E FLUXO DE CO, EM LAVOURA DE SOJA NO RIO GRANDE DO SUL
}

\author{
CELSO PINHEIRO RODRIGUES ${ }^{1}$, DENISE CYBIS FONTANA ${ }^{1}$, OSVALDO LUIZ LEAL DE \\ MORAES $^{2}$, DÉBORA REGINA ROBERTI ${ }^{2}$
}

\author{
${ }^{1}$ Universidade Federal do Rio Grande do Sul (UFRGS), Centro Estadual de Pesquisas em Sensoriamento \\ Remoto e Meteorologia, Porto Alegre, RS, Brasil. \\ ${ }^{2}$ Universidade Federal de Santa Maria (UFSM), Centro de Ciências Naturais e Exatas, Santa Maria, RS, Brasil. \\ py.correio@gmail.com,dfontana@ufrgs.br,osvaldo.moraes@pq.cnpq.br,d_r_roberti@yahoo.com.br
}

Recebido Agosto de 2011 - Aceito Setembro de 2012

\begin{abstract}
RESUMO
O aumento das emissões dos gases de efeito estufa (GEE) se configura, atualmente, como um dos principais problemas ambientais, o que pode afetar significativamente as atividades humanas e os ecossistemas terrestres. Um dos principais GEE é o $\mathrm{CO}_{2}$, o qual tem sido emitido indiscriminadamente em função do estilo de vida atual, assim como pela intensificação das atividades agrícolas. Neste contexto, o objetivo da pesquisa foi estudar a relação entre o comportamento espectral da cultura de soja ao longo de seu ciclo de desenvolvimento, utilizando imagens NDVI (Normalized Difference Vegetation Index) e o fluxo de $\mathrm{CO}_{2}$, calculado pelo método de covariância de vórtices (eddy covariance), gerando informações e metodologia para investigar as trocas de carbono em uma área de cultivo de soja no estado do Rio Grande do Sul, durante a safra de 2008/2009. Utilizou-se imagens TM do satélite Landsat 5, dados fenológicos e dados coletados em estação micrometeorológica ao longo do ciclo de desenvolvimento da soja. Os resultados mostraram que o padrão temporal do fluxo de $\mathrm{CO}_{2}$ ao longo do dia é cíclico, sendo que no período diurno apresenta valores negativos (captura) e no período noturno, positivos (liberação). A radiação solar global determina a magnitude do aprisionamento de $\mathrm{CO}_{2}$ pela cultura da soja, mas o fluxo é modulado pelo estádio fenológico da cultura. A atividade fotossintética das plantas de soja é maior durante o estádio vegetativo, quando coincide a maior incidência de radiação solar e o maior aparato fotossintético. O NDVI, obtido de imagens Landsat, é um indicador da evolução da biomassa da soja ao longo do ciclo. Existe correlação entre o NDVI e o fluxo negativo de $\mathrm{CO}_{2}$ (captura), ocorridos no período diurno. Portanto, técnicas de sensoriamento remoto demonstram potencialidade na geração de informações úteis sobre as trocas de $\mathrm{CO}_{2}$ entre a superfície e a atmosfera. Palavras-Chave: Fluxos de dióxido de carbono, covariância de vórtices, NDVI, soja
\end{abstract}

\begin{abstract}
NDVI AND $\mathrm{CO}_{2}$ FLOW IN A SOYBEAN CROP IN RIO GRANDE DO SUL, BRASIL The increasing on the greenhouse gases (GHG) emissions is today one of the main environmental problems, which can significantly affect human activities and land ecosystems. One of the main GHG is $\mathrm{CO}_{2}$, which has been emitted indiscriminately due to the current lifestyle, as well as the intensification of agricultural activities. In this context, the objective of this investigation was to study the relationship between the spectral behavior of soybean during the crop cycle, using NDVI (Normalized Difference Vegetation Index), and the $\mathrm{CO}_{2}$ fluxes, calculated by the eddy covariance method, generating information and methodology to investigate the carbon exchange in a soybean crop area in the State of Rio Grande do Sul, during the 2008/2009 soybean crop. For this, Landsat images 5 (TM), the phenological information and collected data from micrometeorological station throughout the development cycle of soybean were used. The results showed that the temporal pattern of $\mathrm{CO}_{2}$ flux during the day was cyclical, showing negative values (capture) during daytime and positive values (liberation) at night. The global solar radiation determines the magnitude of the trapping of $\mathrm{CO}_{2}$ by soybean, but the flow is modulated by the phenological stage of the crop. The photosynthetic activity of soybean plants is higher during the vegetative stage, coinciding to the higher incidence of solar radiation and the greater photosynthetic apparatus. The NDVI, obtained from Landsat images,
\end{abstract}


is an indicator of the evolution of soybean biomass during the cycle. NDVI and negative $\mathrm{CO}_{2}$ flow (capture) are correlated during the day. Therefore, remote sensing techniques show potentiality in generating of useful information on $\mathrm{CO}_{2}$ exchange between the surface and atmosphere.

Keywords: $\mathrm{CO}_{2}$ flux, eddy covariance, NDVI, soybean

\section{INTRODUÇÃO}

As mudanças climáticas antropogênicas estão associadas ao aumento da poluição, queimadas, desmatamento, formação de ilhas de calor nas grandes cidades e à atividade agrícola. Segundo o IPCC (2007), estima-se que, das emissões totais, a agricultura contribua com aproximadamente $1 / 3$ da emissão antrópica dos gases do efeito estufa (GEE). O dióxido de Carbono $\left(\mathrm{CO}_{2}\right)$ é o principal gás do GEE. O conhecimento de quanto um ecossistema agrícola contribui para o aumento do dióxido de carbono na atmosfera é um desafio atual e tema de pesquisa de diversos grupos de pesquisa no mundo (Hollinger et al., 2005; Suyker e Verma, 2010; Lopez et al., 2008; Alberto et al., 2009; Sullivan et al., 2010; Zeeman et al., 2010).

As principais técnicas atuais para a estimativa das trocas de $\mathrm{CO}_{2}$ entre um ecossistema e a atmosfera fazem uso de câmaras estáticas ou dinâmicas, perfis de concentração de $\mathrm{CO}_{2}$ no solo ou na atmosfera. Medidas realizadas através de análises de solo ou de câmara são pontuais no tempo e espaço, e podem não capturar a dinâmica dos fluxos de $\mathrm{CO}_{2}$ em diferentes escalas de tempo. A colocação da câmara pode perturbar a integridade da superfície do solo causando uma superestimativa ou subestimativa do fluxo. A técnica de covariância de vórtices (eddy covariance) (Baldocchi et al., 1988), que permite realizar estimativas dos fluxos turbulentos através de medidas de alta frequência de variáveis atmosféricas sem causar perturbação no ambiente, também tem uma delimitação espacial, além de ser necessário o uso de instrumentos caros e de difícil manutenção.

A utilização de técnicas de sensoriamento remoto com o objetivo de fornecer informações sobre o fluxo de $\mathrm{CO}_{2}$, entre a superfície e a atmosfera, ainda não é muito explorada, pois é necessário um estudo da correlação entre as medidas in situ e por sensoriamento remoto. Alguns exemplos são os trabalhos de Paula e Filho (2009), que analisaram a possibilidade de estimar o carbono em florestas naturais; Watzlawick et al. (2009), que realizaram teste de metodologia para estimar a biomassa e carbono orgânico na Floresta com Araucária; Barbosa (2006), que propôs estimar a biomassa e carbono orgânico da parte não inundada da herbácea Echinochloa polystachya. A grande contribuição do sensoriamento remoto está na generalização de informações obtidas em locais específicos para uma grande área, ou seja, na espacialização das mesmas.

$\mathrm{O}$ uso de dados orbitais permite extrair o índice de vegetação por diferença normalizada (NDVI), uma ferramenta disponível para o monitoramento da vegetação. O perfil temporal do NDVI pode ser utilizado para detectar variações sazonais e interanuais na fenologia de culturas, duração do período de crescimento, pico de verde, mudanças fisiológicas das folhas e períodos de senescência (Ponzoni, 2007). Esse índice, portanto, tem sido utilizado com sucesso para o monitoramento de mudanças na vegetação em escala regional, continental e global (Bannari et al., 1995; Huete et al., 2002; Junges e Fontana, 2009). Alguns trabalhos realizados com a cultura da soja no Rio Grande do Sul mostram a utilidade do índice de vegetação na caracterização das respostas das plantas aos fatores do ambiente, assim como, para mapear a área ocupada pela cultura e estimar o rendimento de grãos (Fontana et al., 2003; Jacóbsen et al., 2003; Rizzi, 2005; Wagner et al., 2007; Melo et al., 2008).

O objetivo deste trabalho é correlacionar o NDVI, obtido de imagens de satélite, com os fluxos de $\mathrm{CO}_{2}$ entre a superfície e a atmosfera numa lavoura de soja no noroeste do Rio Grande do Sul. Além disso, serão analisadas as relações entre o NDVI, o fluxo de $\mathrm{CO}_{2}$ e as condições meteorológicas em diferentes etapas do desenvolvimento da cultura.

\section{MATERIAL E MÉTODOS}

\subsection{Sítio experimental}

A área do estudo está situada numa lavoura de dezenas de hectares de cultivo agrícola no município de Cruz Alta-RS, pertencente à CCGL (TEC) - FUNDACEP, fundação privada de pesquisa agropecuária. Nesta lavoura desde 1985 foi instalado um experimento com o objetivo de comparar diferentes manejos do solo combinados com diferentes sistemas de rotação de culturas. A área de interesse é uma fração do terreno da CCGL, a qual é composta por 10 talhões com medidas de $60 \mathrm{~m}$ x $40 \mathrm{~m}$ e 2 talhões com dimensões de $30 \mathrm{~m}$ x $40 \mathrm{~m}$ em um total de 12 talhões. A Figura 1 mostra a composição dos talhões, dividida entre dois manejos: plantio direto (PD), que consiste em plantar espécies sem fazer o revolvimento ou preparo do solo, mantendo cobertura morta ou palha no solo; plantio convencional (PC), que consiste no uso de arado e grade para revolvimento do solo. As medidas usadas neste trabalho são provenientes do talhão PD4 conduzido sob sistema de plantio direto.

Na safra de verão de 2008/2009, o talhão PD4 foi cultivado com soja, cuja semeadura ocorreu no dia 28/11 e a colheita no 
dia 28/04. A cultivar de soja utilizada foi a FUNDACEP 53 RR, com espaçamento entre linhas de $0,40 \mathrm{~m}$. Dados sobre o ciclo de desenvolvimento da cultura foram fornecidos pelos técnicos da CCGL utilizando a escala proposta por Ferh e Cavines (1977).

\subsection{Medidas dos fluxos de $\mathrm{CO}_{2}$}

Uma torre micrometeorológica instrumentada foi instalada no centro do talhão PD4 em 19/11/2008 pela equipe do Laboratório de Micrometeorologia da Universidade Federal de Santa Maria (UFSM), através do projeto SULFLUX ( $h t t p: / /$ www.ufsm,br/sulflux). A localização da torre, no sistema de projeção UTM (Universal Transversa de Mercator), datum WGS84, foi nas coordenadas 238728, 6833336, obtida com um GPS Garmin modelo 76CSx e antena externa, com sinal de 12 satélites. Os fluxos de $\mathrm{CO}_{2}$ foram estimados pelo método de covariância dos vórtices através das medidas na frequência de $10 \mathrm{~Hz}$, da componente vertical da velocidade do vento, obtidas por um anemômetro sônico (CSAT 3, Campbell Scientific Inc), e da concentração de $\mathrm{CO}_{2}$, obtidas por um analisador de gás infravermelho (LiCor, LI7500), ambos localizados a 2,5 m. Da torre micrometeorológica foram utilizados dados dos sensores (altura do sensor na torre, fabricante, modelo) com medidas realizadas na frequência de $10 \mathrm{~Hz}$ : (vento e temperatura do ar (2,5 m, CSAT 3, Campbell Scientific Inc., Logan, UT); $\mathrm{H}_{2} \mathrm{O} / \mathrm{CO}_{2}$ analisador de gás e pressão (2,5 m, LiCor, LI7500); radiação de ondas curtas incidente (5 m, Kipp \& Zonen, CMB6); radiação fotossinteticamente ativa incidente (PAR) (1 m, Kipp \& Zonen, PAR LITE); e precipitação pluvial (1,5 m, Texas Instruments). Mais detalhes em Roberti et al. (2011).

\subsection{Imagens orbitais}

Foram utilizadas imagens TM do Satélite Landsat 5, como referência a projeção UTM (Universal Transverse Mercator), o datum WGS/84 (World Geodetic System - 1984, Zone 22), órbita/ponto 223/080 com as datas (entre parênteses o dia Juliano, DJ, respectivo): 20 de dezembro de 2008 (DJ =355), 05 de janeiro de 2009 (DJ =5), 21 de janeiro de 2009 (DJ =21), 26 de março de 2009 (DJ =26) e 11 de abril de 2009 (DJ =101), para gerar as imagens NDVI e os perfis temporais. Foi também utilizada imagem do CBERS 2B instrumento HRC, órbita/ponto $160 \_$D/132_3 de 25 de novembro de 2008, para delimitar os talhões. As datas para análise foram escolhidas levando em consideração a menor ocorrência de nuvens e disponibilidade dos dados da torre micrometeorológica.

No intuito de caracterizar a resposta espectral da cultura da soja de forma multitemporal, foi efetuada a calibração radiométrica, atmosférica e geométrica nas imagens Landsat (Rodrigues, 2010) e geradas imagens de índices de vegetação
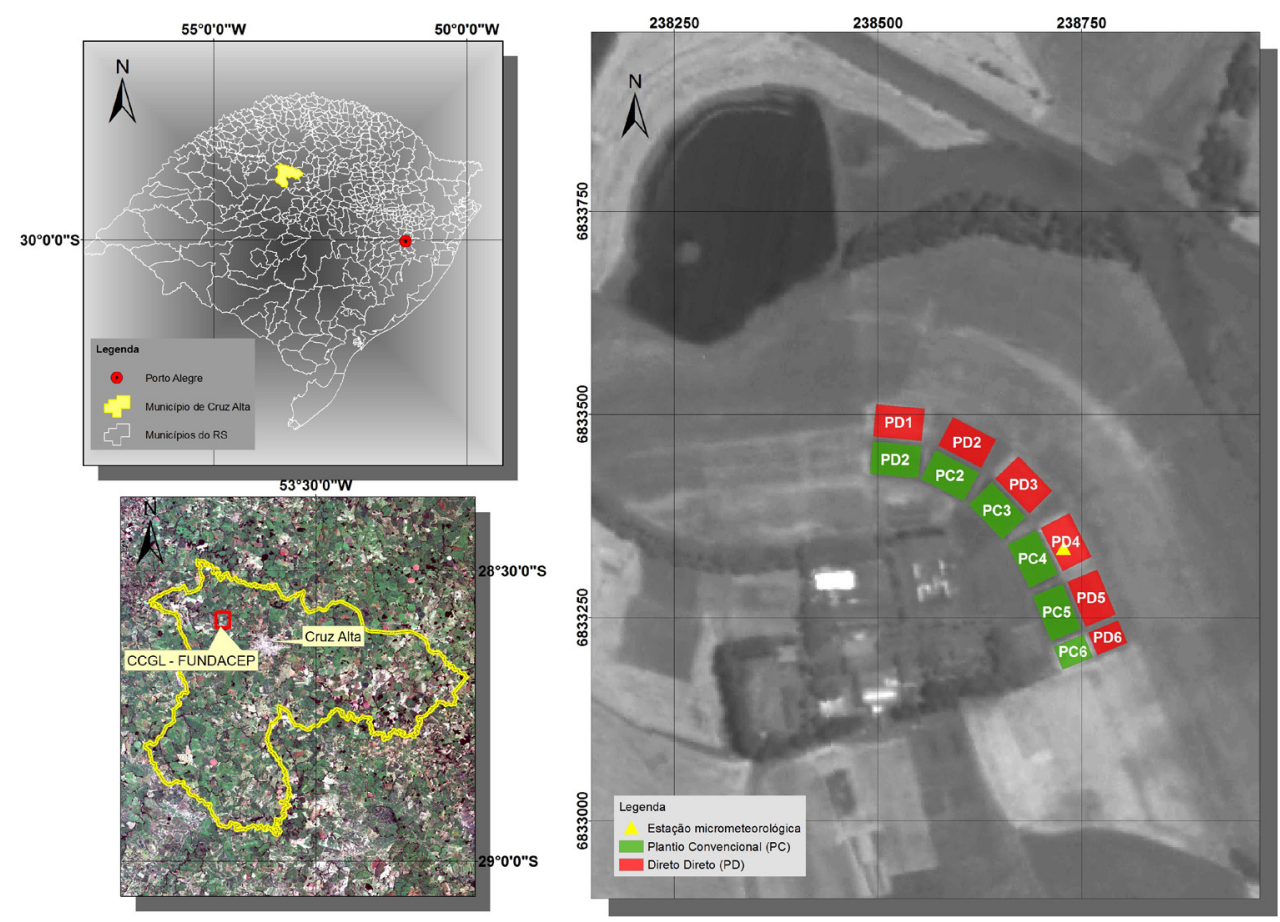

Figura 1. Situação do município de Cruz Alta no estado do Rio Grande do Sul e localização da área da CCGL (TEC) - FUNDACEP no município de Cruz Alta na imagem TM - Landsat 5 de 21/01/09 em composição RGB 321, dos talhões e da estação micrometeorológica na área experimental sobre a imagem do CBERS 2B instrumento HRC de 25/11/08 na banda pancromática. 
por diferença normalizada - NDVI (Rouse et al., 1973), através da equação:

$$
N D V I=\frac{\left(\rho_{i}-\rho_{v}\right)}{\left(\rho_{i}+\rho_{v}\right)}
$$

onde NDVI é o índice de vegetação por diferença normalizada, do inglês Normalized Difference Vegetation Index; $\rho_{\dot{v}}$ e $\rho_{v}$ são as reflectâncias no infravermelho próximo e no vermelho (bandas 4 e 3 do sensor TM - Landsat 5, respectivamente).

A imagem de NDVI foi gerada usando o módulo Vegindex do SIG Idrisi, e, após, foi localizada a estação micrometeorológica e extraídos os dados de NDVI sobre a mesma, utilizando uma janela de amostragem de dois pixels.

Criou-se uma superfície de absorção de $\mathrm{CO}_{2}$ atmosférico pela lavoura de soja através do modelo TIN (Triangulated Irregular Network), a partir da interpolação dos valores de fluxo de $\mathrm{CO}_{2}$ nos dias em que a área experimental foi imageada pelo satélite. Por fim, analisou-se a correlação entre o fluxo de $\mathrm{CO}_{2}$, obtidos da torre micrometeorológica, e os dados de NDVI, obtidos das imagens, utilizando os seguintes indicadores: Inst - o valor instantâneo do fluxo de $\mathrm{CO}_{2}$ no horário da passagem do Satélite (10h:15min), Med $2 \mathrm{~h}$ - o valor da média do fluxo de $\mathrm{CO}_{2}$ de duas horas centrada no horário da passagem; Med negat - a média de todo o período em que o fluxo de $\mathrm{CO}_{2}$ é negativo; Med 24h - a média do fluxo de $\mathrm{CO}_{2}$ das 24h; Área - integração da curva do período em que o fluxo de $\mathrm{CO}_{2}$ é negativo; Perímetro - o perímetro da curva na período em que o fluxo de $\mathrm{CO}_{2}$ é negativo; Pico negat - o valor máximo de fluxo do $\mathrm{CO}_{2}$ no período em que o fluxo é negativo.

Para os dias com ausência de nebulosidade foi feito o ajuste de uma equação de regressão linear, através do método dos mínimos quadrados e testada a significância da mesma. Nas análises foram utilizados os softwares Excel e AutoCAD MAP.

\section{RESULTADOS E DISCUSSÃO}

\subsection{Condições Meteorológicas}

A Figura 2 apresenta a variação diária da radiação solar global (Rg), da radiação fotossinteticamente ativa (PAR), da temperatura do ar e da pressão atmosférica para as datas consideradas neste trabalho, dias julianos 355 de 2008 e 5, 21, 85 e 101 de 2009. Como o critério de escolha selecionou as datas que apresentavam a menor ocorrência de nuvens. Nota-se que praticamente todos os dias avaliados apresentaram um padrão diurno similar para as variáveis atmosféricas. A PAR representou em média $50 \%$ de Rg. O máximo valor diário de Rg e PAR ocorreu próximo das $12 \mathrm{~h}: 00 \mathrm{~min}$, sendo superior nos dias julianos 355, 5 e 21, com valores de Rg superiores a 1.100 $\mathrm{Wm}^{-2}$. Nos dias 85 e 101 os valores de $\mathrm{Rg}$ foram inferiores a
$900 \mathrm{Wm}^{-2}$. Nos dias 355 e 85 as curvas mostraram diminuições abruptas no recebimento de $\mathrm{Rg}$ e PAR no período da tarde, o que foi consequência da nebulosidade.

Associado ao padrão da radiação solar global, verificou-se que as temperaturas do ar (Figura 2C) tiveram valores menores durante a noite, mínimo próximo às $7 \mathrm{~h}: 00 \mathrm{~min}$, e maiores durante o dia. O horário de ocorrência dos máximos valores variou conforme o dia de observação, mas em geral, foi após às $13 \mathrm{~h}: 00 \mathrm{~min}$. Este padrão de variação diária da temperatura é típico de dias em que o aquecimento e resfriamento do solo e do ar são definidos pelas trocas entre a superfície e a atmosfera. .

Ao longo dos dias analisados, a pressão atmosférica mostrou uma associação aproximadamente inversa com a temperatura do ar, com os dias mais frios apresentando pressão atmosférica maior. Já ao longo do dia, a pressão mostrou dois máximos e dois mínimos, o que em geral é observado e denominado como marés barométricas. Novamente os dias 355 e 85 se diferenciam dos demais por apresentarem pressões atmosféricas inferiores (Figura 2D).

A direção e intensidade do vento para os dias considerados são apresentados na Figura 3. Nos dias 5, 21 e 101 os ventos de noroeste predominaram, atingindo as maiores velocidades às $8 \mathrm{~h}: 44 \mathrm{~min}, 21 \mathrm{~h}: 27 \mathrm{~min}$ e $20 \mathrm{~h}: 06 \mathrm{~min}$, respectivamente. Já nos dias 355 e 85 os ventos apresentaram comportamento mais variável ao longo do dia, com ventos no quadrante norte, sul e sudoeste durante o dia e ventos no quadrante noroeste e sudoeste durante a noite, atingindo as maiores velocidades na madrugada e no entardecer.

Destas análises observa-se, portanto, que os dias 355 e 85 apresentaram uma dinâmica distinta dos demais. A radiação solar global sofreu alterações abruptas no período da tarde que iniciou próximo das $17 \mathrm{~h}$ no dia 355 e das $13 \mathrm{~h}$ no dia 85 , retornando mais ao final da tarde ao padrão dos outros dias. Essas oscilações estão ligadas provavelmente pela presença de nuvens (Figura 2A). Também nestes dias ocorreu grande oscilação na temperatura do ar (Figura 2C) e na direção dos ventos (Figura 3), o que indica a entrada de um sistema frontal, ocasionando chuvas nos dias posteriores.

\subsection{NDVI}

A Tabela 1 mostra os valores de NDVI e os estádios fenológicos da cultura da soja ao longo do período experimental. A análise dos dados mostrou que existe associação entre estes dois conjuntos de dados.

Do estádio V2 ao V9, houve aumento da biomassa verde, o qual foi acompanhado por incrementos nos valores do NDVI. O máximo NDVI coincidiu com o estádio V9, quando a cultura apresentou o máximo vigor vegetativo. Após o máximo, levando em conta as imagens disponíveis, iniciou-se o declínio 

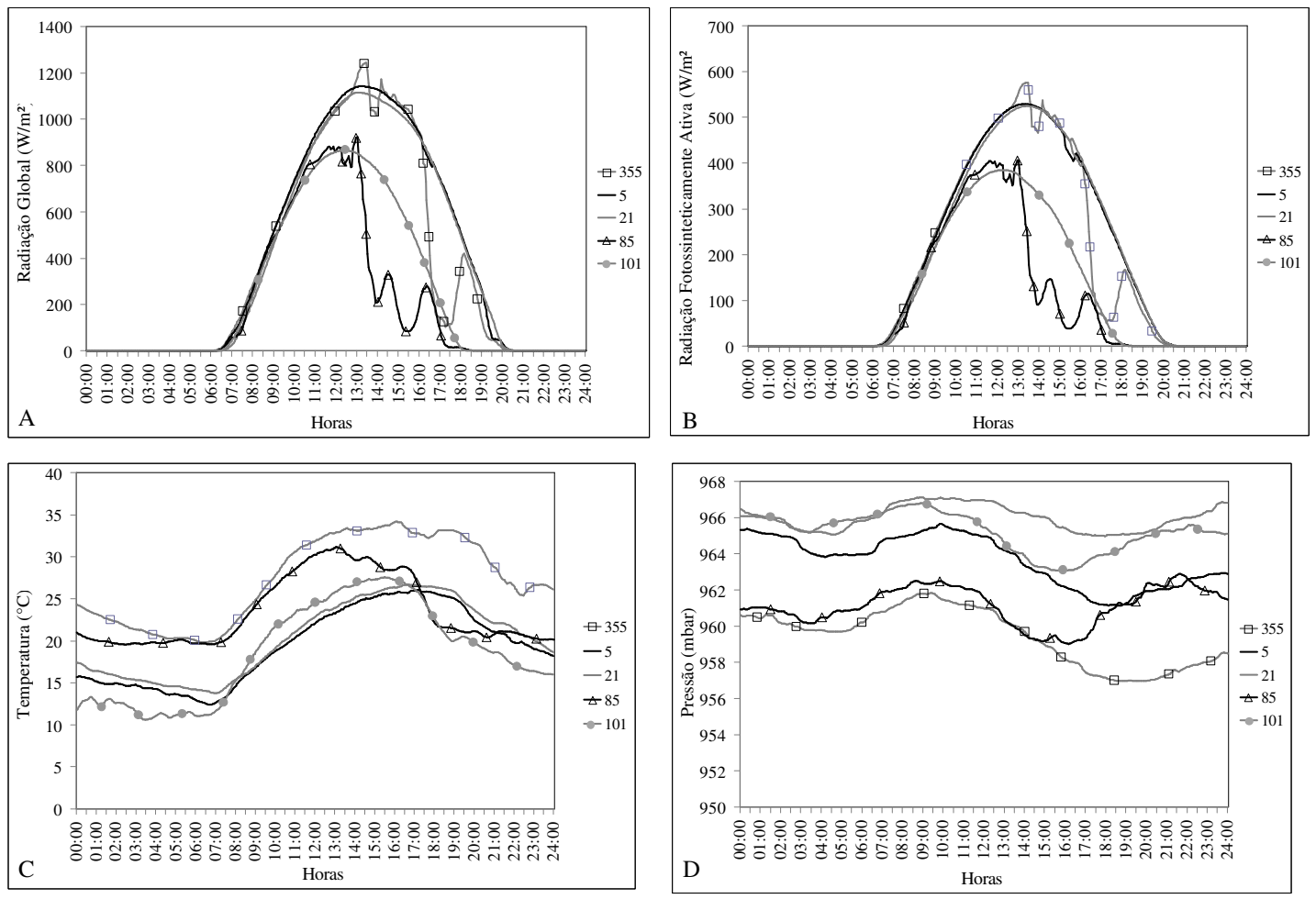

Figura 2 - Variações diária da Radiação Solar Global (A), da Radiação Fotossinteticamente Ativa (B), da Temperatura do ar (C) e da Pressão Atmosférica (D) para os dias julianos 355 de 2008 e 5, 21, 85 e 101 de 2009.

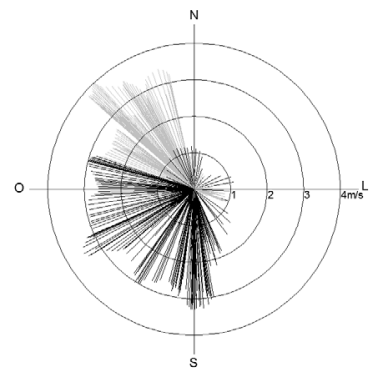

Dia juliano 355

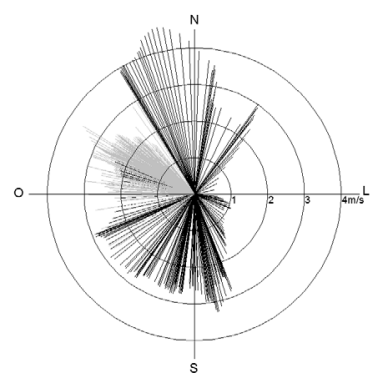

Dia juliano 85

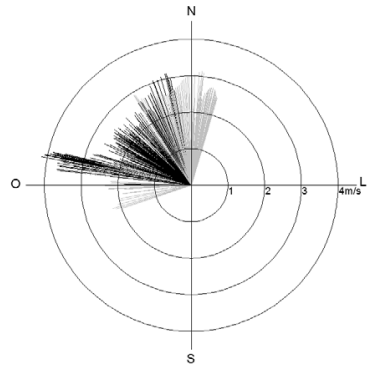

Dia juliano 5

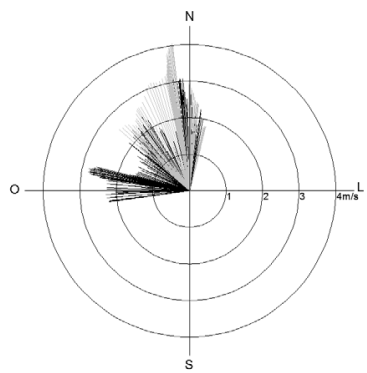

Dia juliano 101

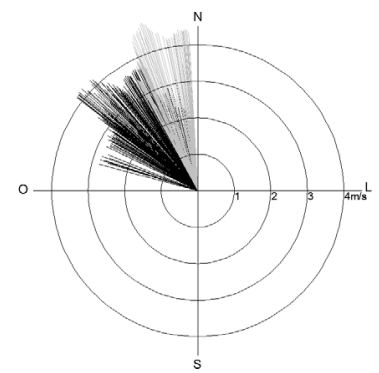

Dia juliano 21

Figura 3 - Variação diária da Velocidade e Direção do Vento ao longo do período experimental de 2008/09. As linhas pretas representam o período diurno e as cinzas o noturno. 
Tabela 1 - Índice de Vegetação por diferença Normalizada (NDVI) e estádios fenológicos da soja durante o período experimental (2008/09) Fonte: (EMBRAPA SOJA, 2005).

\begin{tabular}{ccccc}
\hline $\begin{array}{c}\text { Data do } \\
\text { imageamento }\end{array}$ & $\begin{array}{c}\text { Dia } \\
\text { Juliano }\end{array}$ & NDVI & Estádio & Descrição \\
\hline $20 / 12 / 08$ & 355 & 0,596 & V2 & $\begin{array}{c}\text { Pequeno acúmulo de biomassa no início do } \\
\text { ciclo. }\end{array}$ \\
$05 / 01 / 09$ & 5 & 0,787 & V5 & Crescimento da cultura. \\
$21 / 01 / 09$ & 21 & 0,840 & V9 & Máximo vigor da cultura. \\
$26 / 03 / 09$ & 85 & 0,733 & R7.1 & Folhas começam a amarelar e cair. \\
$11 / 04 / 09$ & 101 & 0,402 & R9 & Ponto de maturação da colheita \\
\hline
\end{tabular}

dos valores do NDVI, que acompanharam o ciclo evolutivo e a diminuição do vigor da cultura, até a mesma finalizar o ciclo. No ponto de maturação o NDVI foi aproximadamente $50 \%$ do valor máximo obtido neste estudo.

\subsection{Fluxo de $\mathrm{CO}_{2}$}

O ciclo diário dos fluxos de $\mathrm{CO}_{2}$ para a soja nas datas do imageamento é mostrado na Figura 4. Os valores positivos são definidos como emissão de $\mathrm{CO} 2$ e negativos como absorção de $\mathrm{CO}_{2}$. Os diferentes estádios fenológicos estão bem caracterizados nas datas escolhidas, mostrando maiores valores da absorção diurna e emissão noturna nos estádios de crescimento (V5) e máximo vigor da cultura (V9) e menores valores estádio do ponto de maturação (R9). É importante notar que no DJ 85 ocorre a emissão de $\mathrm{CO}_{2}$, nos horários da tarde, diferindo muito do comportamento dos demais dias analisados. Isto deve-se ao fato de uma queda abrupta no valor do PAR a partir das $13 \mathrm{~h}$ (conforme Figura 2b). O mesmo comportamento ocorre para o DJ 355, no entanto neste dia a PAR teve uma diminuição apenas entre as 17 e 18h, o que acarretou numa emissão de $\mathrm{CO}_{2}$ neste horário.

Os valores de emissão e absorção de $\mathrm{CO}_{2}$ obtidos para a soja neste experimento estão de acordo com os valores encontrados por Verma et al.(2005) e Hollinger et al. (2005), cujos experimentos foram realizados na região central americana.

Figura 5 ilustra a associação entre a absorção de $\mathrm{CO}_{2}$ atmosférico e a disponibilidade de radiação para o processo fotossintético da cultura da soja, evidenciando a alta associação entre estas duas variáveis. A radiação fotossinteticamente ativa (PAR) teve uma diminuição contínua ao longo dos dias de observação, o que é função da declinação solar. Já o fluxo de $\mathrm{CO}_{2}$ foi determinado pela disponibilidade de radiação, mas modulado pelas condições da superfície, especialmente da densidade de biomassa. $\mathrm{O}$ valor mais elevado de fluxo negativo foi encontrado no dia 21/01/09, quando a cultura estava no estádio V9 (Tabela 1). Neste estádio, com todo o aparato

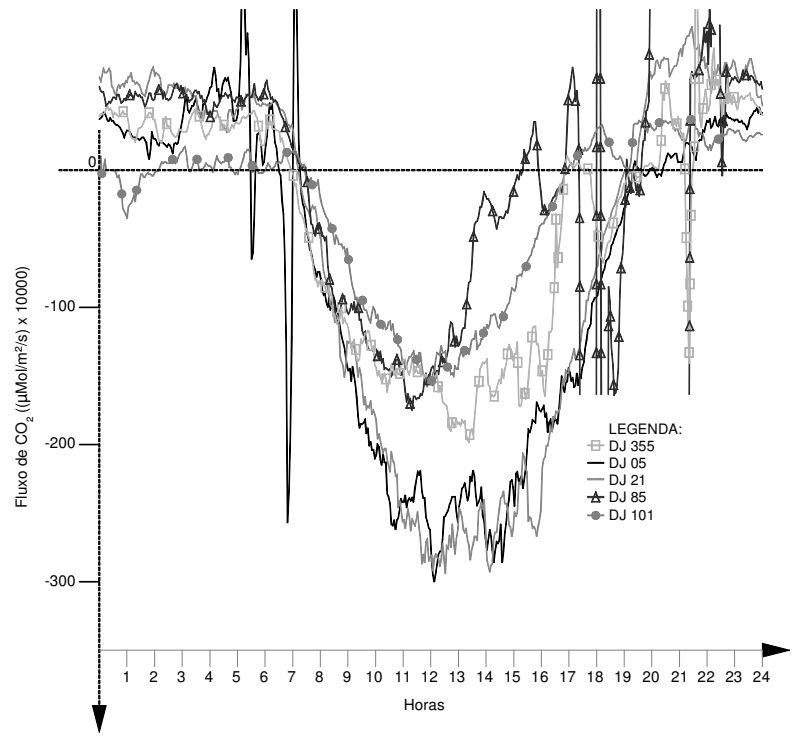

Figura 4 - Variação do fluxo de $\mathrm{CO}_{2}$ ao longo das 24 horas para os dias julianos 355, 05, 21, 85 e 101 (2008/09).

fotossintético já desenvolvido e com alta disponibilidade de radiação solar, a planta de soja capturou a maior quantidade de $\mathrm{CO}_{2}$ da atmosfera. $\mathrm{O}$ menor valor de fluxo de $\mathrm{CO}_{2}$ foi observado no dia 355 , quando apesar da alta disponibilidade de radiação solar, havia uma pequena quantidade de biomassa (estádio V2). Já nos dias 85 e 101, apesar da cultura se encontrar com alta biomassa, respectivamente, nos estádios R7.1 e R9, a disponibilidade de radiação era menor, determinando fluxos menores. Em linhas gerais, o fluxo de $\mathrm{CO}_{2}$ apresentou um padrão diurno gradual, onde aumentou a partir do nascer do sol até o meio dia e decresceu no final da tarde. Nos dias 355 e 85, quando a radiação incidente sofreu alterações no final do dia, percebeu-se as mesmas oscilações também no fluxo de $\mathrm{CO}_{2}$ (Figura 5), condizente com o estudo de Soegaard et al. (2003), que estudaram o fluxo de $\mathrm{CO}_{2}$ em culturas de grãos na Dinamarca, verificando menores taxas em dias nublados.

Em todos os parâmetros avaliados, observou-se que o dia 26/03/09 (DJ 85) foi caracterizado por um padrão 


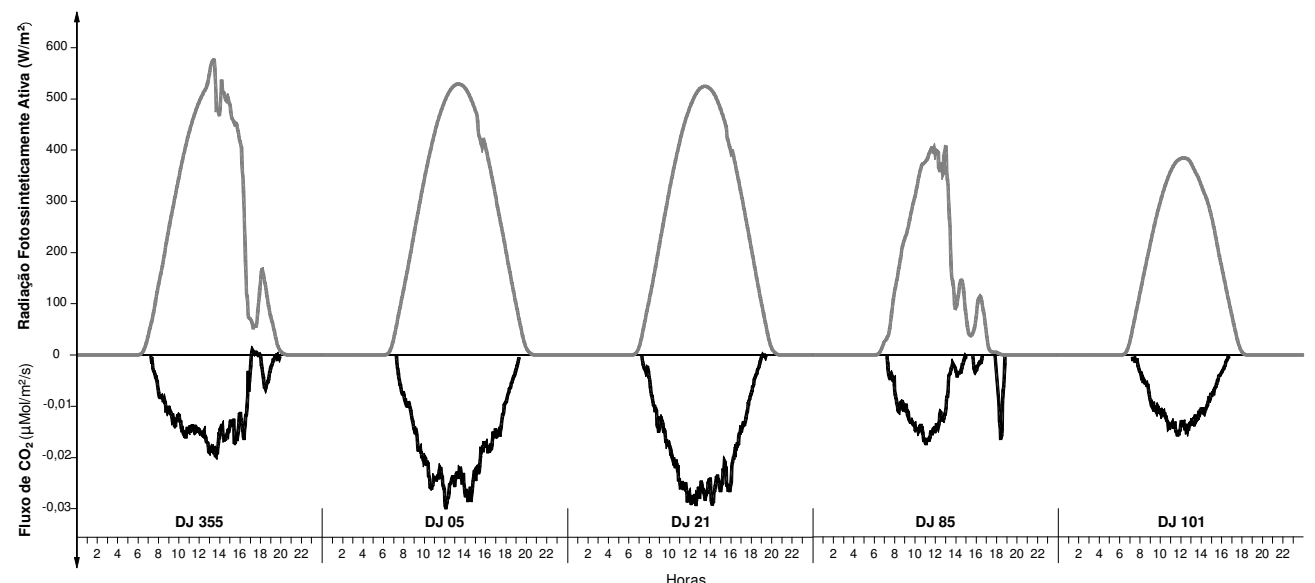

Figura 5 - Variações da Radiação Fotossinteticamente Ativa (PAR) e fluxo de $\mathrm{CO}_{2}$ nos dias julianos 355, $05,21,85$ e 101.

distinto dos demais. Percebe-se que nesta data as condições micrometeorológicas apresentaram oscilações importantes, são elas: diminuição abrupta da radiação solar global, queda de temperatura do ar, aumento de pressão atmosférica e variação na velocidade e direção do vento.

$\mathrm{Na}$ Figura 6 é representada a superfície de carbono, a qual integra algumas das variáveis implicadas no estudo da absorção de carbono. Pode-se observar que o fluxo de $\mathrm{CO}_{2}$ atingiu os maiores valores (cor verde na figura) quando ocorreu o máximo de disponibilidade de energia e o máximo de capacidade de absorção pela superfície.

No que tange a radiação solar, nota-se, como é esperado, que quanto mais próximo do meio dia, maiores são os valores de assimilação de carbono. No estádio vegetativo, também ocorre o mesmo, pois é nesse período que a cultura está construindo o aparelho fotossintético. Nota-se que no estádio R7.1 a incidência solar menor acarreta valores mais baixos de $\mathrm{CO}_{2}$, somando-se a isso a possibilidade da presença de nuvens, menores valores em módulo foram encontrados.

\subsection{Correlações entre NDVI e fluxo de $\mathrm{CO}_{2}$}

Na Tabela 2 são apresentados os coeficientes de correlação obtidos entre o NDVI e os dados derivados da curva do fluxo de $\mathrm{CO}_{2}$, os quais estão descritos no item 2.3.

Os coeficientes de correlações apresentaram valores negativos e positivos, oscilando entre um mínimo de -0,5063 e um máximo de 0,8151 . Os valores negativos indicam uma relação inversa, o que é esperado, pois quanto maior o valor do NDVI, maior a biomassa, maior a potencialidade de captura de $\mathrm{CO}_{2}$ através do processo fotossintético, maior é a absorção de $\mathrm{CO}_{2}$ (fluxo de $\mathrm{CO}_{2}$ negativo) pela cultura de soja.

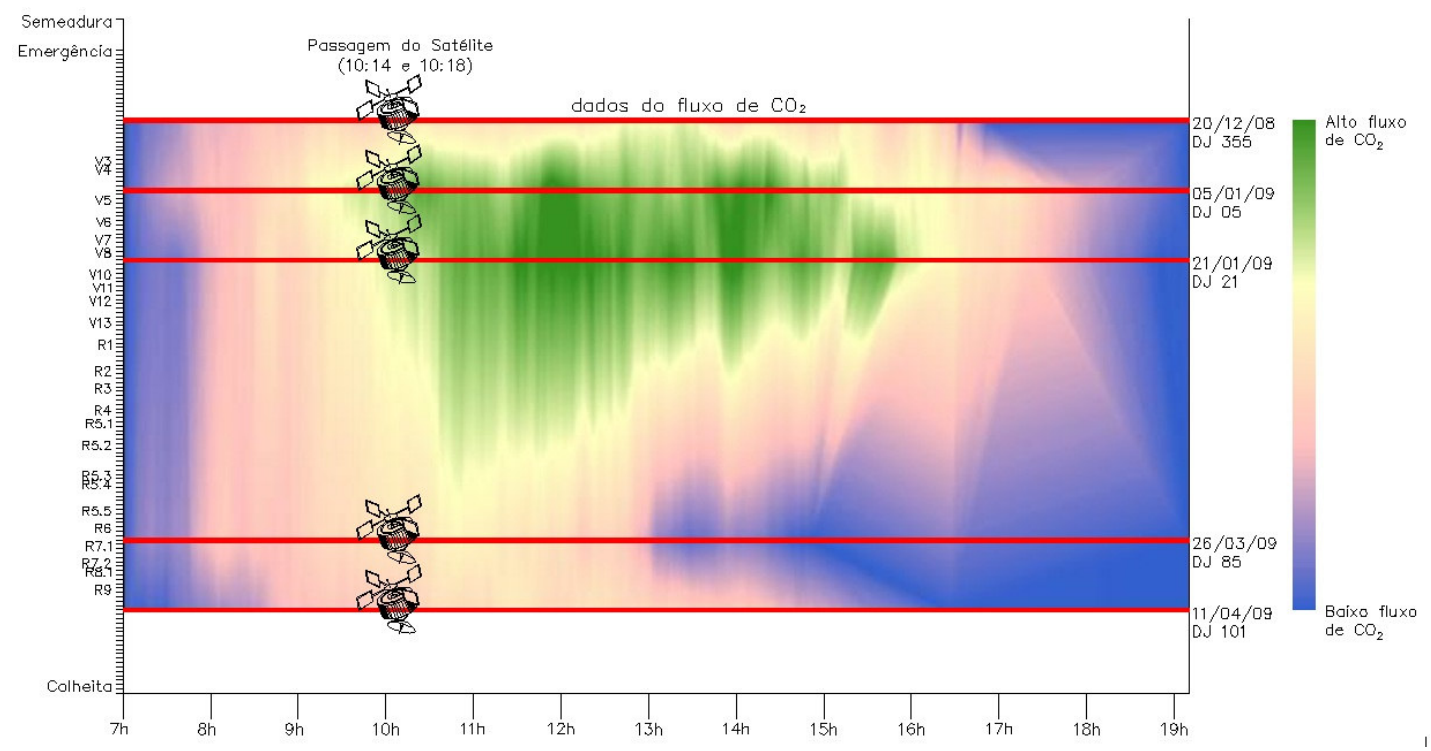

Figura 6 - Superfície de fluxo de carbono para a cultura da soja no período experimental de 2008/09. 
Tabela 2 - Valores observados do Índice de Vegetação por diferença normalizada (NDVI) e dos parâmetros obtidos da curva de fluxo diário de $\mathrm{CO}_{2}$ atmosférico $\left(\mu \mathrm{Molm}^{-2} \mathrm{~s}^{-1}\right.$ ) para os dias julianos (DJ) 355, 5, 21, 85 e 101 e os coeficientes de correlação entre NDVI e fluxo de $\mathrm{CO}_{2}$ sobre uma lavoura de soja.

\begin{tabular}{llccccc}
\hline Parâmetro & DJ 355 & DJ 5 & DJ 21 & DJ 85 & DJ 101 & $\begin{array}{c}\text { Coeficiente } \\
\text { de correlação }\end{array}$ \\
\hline NDVI & 0,5967 & 0,7877 & 0,8405 & 0,7337 & 0,4022 & \\
& & & & & & \\
Inst & $-0,0192$ & $-0,0234$ & $-0,0261$ & $-0,0089$ & $-0,0133$ & $-0,5440$ \\
Med 2h & $-0,0172$ & $-0,0249$ & $-0,0270$ & $-0,0081$ & $-0,0129$ & $-0,5690$ \\
Med negat & $-0,0105$ & $-0,0177$ & $-0,0174$ & $-0,0262$ & $-0,0091$ & $-0,6540$ \\
Med 24 h & $-0,0039$ & $-0,0076$ & $-0,0059$ & $-0,0140$ & $-0,0029$ & $-0,5060$ \\
Pico negat & $-0,0198$ & $-0,0300$ & $-0,0294$ & $-0,0175$ & $-0,0158$ & $-0,7850$ \\
Área ${ }^{1,2,3}$ & 26572 & 42518 & 42484 & 18695 & 17254 & 0,7570 \\
Perímetro $^{1,2,3}$ & 1558 & 1701 & 1756 & 2199 & 890 & 0,8150 \\
\hline
\end{tabular}

Inst - é o valor instantâneo do fluxo de $\mathrm{CO}_{2}$ do horário da passagem do Satélite (10h:15min), Med $2 \mathrm{~h}$ - é o valor da média do fluxo de $\mathrm{CO}_{2}$ de duas horas centrada no horário da passagem; Med negat - é a média do fluxo de $\mathrm{CO}_{2}$ de todo o período em que o fluxo é negativo; Med 24h - é a média do fluxo de $\mathrm{CO}_{2}$ das $24 \mathrm{~h}$; Área - é integração da curva do fluxo de $\mathrm{CO}_{2}$ do período em que o fluxo é negativo; Perímetro - é o perímetro da curva do fluxo de $\mathrm{CO}_{2}$ no período em que o fluxo é negativo; Pico negat - é o valor máximo da curva do fluxo de $\mathrm{CO}_{2}$ no período em que o fluxo é negativo.

${ }^{1}$ Valores multiplicados por 10000 .

${ }^{2}$ Correlações significativas a $90 \%$ de probabilidade

${ }^{3}$ Obtidos das seções no período em que o fluxo é negativo

Correlações positivas ocorrem somente quando se utilizou a integração da curva (área) e o perímetro da curva, as quais aliadas ao parâmetro "pico negativo", foram as relações com coeficientes de correlação mais elevados e significativos estatisticamente. Dado o pequeno número de pares de dados usados nesta análise (5), somente os coeficientes de correlação superiores a 0,7 são significativos a $90 \%$ de probabilidade.

A evolução temporal do NDVI da lavoura de soja, ao longo do período experimental (Figura 7), é caracterizada por baixos valores no início da cultura, um gradativo aumento até chegar à etapa de máximo acúmulo da biomassa e novamente retornando a valores mais baixos na etapa de senescência, percorrendo todos os estádios de desenvolvimento. Considerando as imagens disponíveis, o maior valor de NDVI ocorreu na data de 21/01/2009 (Dia Juliano 21). Evolução temporal do NDVI, sobre áreas de soja similar a descrita, pode ser encontrada em trabalhos realizados no Rio Grande do Sul, que utilizaram sensores com alta resolução temporal como o AVHRR/NOAA (Jacobsen et al., 2003; Melo et al., 2008) ou MODIS/Terra (Wagner et al., 2007) para monitorar a evolução da cultura. Estes autores verificaram que existe uma importante variabilidade interanual das curvas de NDVI ao longo do ciclo, o que está em grande parte associada às condições meteorológicas. Neste trabalho, mesmo usando imagens Landsat, foi possível acompanhar a evolução da cultura, dada a disponibilidade de cinco imagens sem cobertura de nuvem.

Ainda na Figura 7 pode-se observar o transcurso temporal dos diferentes parâmetros obtidos da curva do $\mathrm{CO}_{2}$. Os parâmetros designados por pico de $\mathrm{CO}_{2}$ e área, além de apresentarem coeficientes de correlação significativos, apresentaram, também, uma curva com forma similar a da curva temporal do NDVI (Figura 7A e 7B). Ambos os parâmetros representam uma evolução temporal adequada à capacidade de absorção de $\mathrm{CO}_{2}$ pela superfície, especialmente pela biomassa
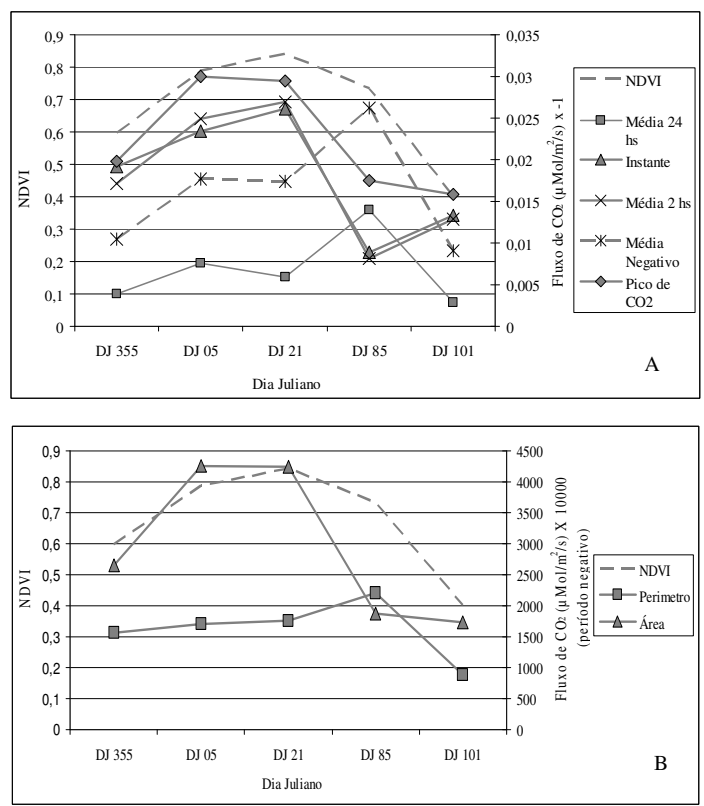

Figura 7 - Evolução temporal do NDVI e dos parâmetros obtidos da curva do fluxo de $\mathrm{CO}_{2}$ : média das 24 horas, instante da passagem do satélite, média de 2 horas, médias do fluxo negativo e pico de $\mathrm{CO}_{2}$, nos dias julianos 355, 5, 21, 85 e 101 (A) e Evolução temporal do NDVI e dos parâmetros obtidos da curva do fluxo de $\mathrm{CO}_{2}$ : área e perímetro, nos dias julianos 355, 5, 21, 85 e 101 (B). 
da cultura da soja, o que é caracterizado pelo NDVI. Apesar do maior coeficiente de correlação ter sido obtido entre o NDVI e o perímetro da seção (Figura 7B), o padrão das curvas não foi coincidente e a explicação física desta relação não é evidente.

Em todos os parâmetros avaliados observou-se que o dia 26/03/09 (DJ 85), foi caracterizado por um padrão distinto do esperado. As condições micrometeorológicas diferenciadas podem ter interferido na fisiologia das plantas ocasionando o fechamento dos estômatos. Também se deve considerar que em condições de experimentação de campo, os sensores da torre estão expostos a situações que podem em certas circunstâncias alterar as medições como, por exemplo, insetos, partículas d água, etc.

Caso no DJ85 tivesse sido observada uma evolução diária do fluxo de $\mathrm{CO}_{2}$, semelhante a dos demais dias de observação, os valores das correlações seriam bem maiores, podendo levar a equivocada conclusão de que o fluxo de $\mathrm{CO}_{2}$ é determinado basicamente pela densidade de biomassa verde na superfície. Este dia, portanto, é extremamente didático, visto que ele explicita a influência de outros fatores do ambiente na determinação da magnitude do fluxo de $\mathrm{CO}_{2}$, além da biomassa.

Apesar disso, pode-se ajustar uma função para estimar o fluxo de $\mathrm{CO}_{2}$ (variável de medição localizada e onerosa) a partir de dados de NDVI (variável de medição regional e baixo custo) somente para dias com ausência de nebulosidade persistente. Abaixo são apresentadas as equações para os dois parâmetros da curva de $\mathrm{CO}_{2}$, que apresentaram correlação significativa e explicação física consistente.

$$
\begin{aligned}
& \mathrm{CO}_{2} \text { Pico_negat }=-0,034 \mathrm{NDVI}-0,001 \\
& \mathrm{R}^{2}=0,948 \\
& \mathrm{CO}_{2} \text { Área }=61881 \mathrm{NDVI}-8435 \\
& \mathrm{R}^{2}=0,977
\end{aligned}
$$

onde $\mathrm{CO}_{2}$ Pico_negat é expresso em $\mu \mathrm{Mol} / \mathrm{m}^{2} / \mathrm{s}$ e $\mathrm{CO}_{2}$ Area é expresso em $\left(\mu \mathrm{Mol} / \mathrm{m}^{2} / \mathrm{s}\right) * 10000$.

Ressalta-se que as equações 2 e 3 foram ajustadas com um número muito reduzido de dados (somente 4). Entretanto, este tipo de equação somente é possível ser ajustada para dias ensolarados, pois em dias com cobertura de nuvens não é possível obter informação sobre o NDVI a partir de imagens Landsat. $\mathrm{O}$ ajuste destas funções, mesmo com as limitações existentes, é importante porque sinaliza a possibilidade de em trabalhos futuros fazer a validação das mesmas.

\section{CONCLUSÕES}

Neste trabalho foram estimadas as correlações entre trocas de $\mathrm{CO}_{2}$, obtidas pelo método eddy covariance e o NDVI obtido de sensoriamento remoto numa cultura de soja no Sul do Brasil.
A disponibilidade de radiação solar determina a magnitude do aprisionamento de $\mathrm{CO}_{2}$ pela cultura da soja, mas o fluxo é modulado pelo estádio fenológico da cultura. A atividade fotossintética das plantas de soja é maior durante o estádio vegetativo, quando coincide a maior incidência de radiação solar e o maior aparato fotossintético.

O NDVI, obtido de imagens Landsat, é um indicador da evolução temporal da biomassa verde da soja ao longo do ciclo; apresenta baixos valores no início do ciclo, cresce gradualmente até um máximo de biomassa e decresce com o término do ciclo da cultura.

Existe correlação entre o NDVI e a absorção de $\mathrm{CO}_{2}$ num cultivo de soja , no período diurno. Para dias com ausência de nebulosidade persistente é possível ajustar funções de estimativa entre estes dois indicadores. Portanto, técnicas de sensoriamento remoto demonstram potencialidade na geração de informações quantitativas sobre as trocas de $\mathrm{CO}_{2}$ entre a superfície e a atmosfera.

\section{REFERÊNCIAS BIBLIOGRÁFICAS}

ALBERTO, M. C. R.; WASSMANN, R.; HIRANO, T.; et al. $\mathrm{CO} 2$ /heat fluxes in rice fields: Comparative assessment of flooded and non-flooded fields in the Philippines. Agricultural and Forest Meteorology, v.149, p.17371750, 2009.

BALDOCCHI, D.D., Hicks, B.B., Meyers, T.P. Measuring biosphere-atmosphere exchanges of biologically related gases with micrometeorological methods. Ecology 69, 1331-1340, 1988.

BANNARI, A. MORIN, D.;BONN, F.; HUETE, A. R. A review of vegetation indices. Remote Sensing Reviews, v.13, p. 95-120, 1995.

BARBOSA, K.M.N. Monitoramento espacial de biomassa carbono orgânico da vegetação herbácea de várzea na Amazônia Central. 2006. 131p. Tese (Doutorado em Ciências Florestais). Programa de Pós-Graduação em Engenharia Florestal, Setor de Ciências Agrárias, Universidade Federal do Paraná, Curitiba, 2006.

EMBRAPA. Empresa Brasileira de Pesquisa Agropecuária. Soja: 2005. Disponível em: <www.cnpso.embrapa.br/ producaosojaPR/SojanoBrasil $>$ Acessado em Maio. 2010.

FEHR, W. R.; CAVINESS, C. E. Stages of soybean development. Ames: State University of Science and Technology (Special Report, 80), 11 p. 1977.

FONTANA, D.C.; WEBER, E.; DUCATI, J.R.; BERLATO, M.A.; GUASSELLI, L.A.; GUSSO, A. Monitoramento da cultura da soja no centro-sul do Brasil durante a La Niña 1998/2000. Revista Brasileira de Agrometeorologia, Santa Maria, v. 10, n.1, p.135-145. 2003. 
HOLlingER, S. E., BERNACCHI, C. J., MEYERS, T. P. Carbon budget of mature no-till ecosystem in North Central Region of the United States. Agricultural and Forest Meteorology, v.130, p. 59-69, 2005.

HUETE, A.R.; DIDAN, K.; MIURA, T. et al. Overview of the radiometric and biophysical performance of the MODIS vegetation index. Remote Sensing of Environment, v.83, p.195-213, 2002.

IPCC. Intergovernmental Panel on Climate Change. Climate Change 2007: The Physical Science Basis. SOLOMON, S. D.; QIN, M.; MANNING, Z.; CHEN, M.; MARQUIS, K. B.; AVERYT, M. (eds.). Cambridge University press, Cambridge, 2007. 996 p.

JACÓBSEN, L. O.; FONTANA, D. C.; SHIMABUKURO, Y. E. Alterações na vegetação em macrozonas do Rio Grande do Sul associadas a eventos El Niño e La Niña, usando imagens NOAA. Revista Brasileira de Agrometeorologia, v.11, p.361 - 374, 2003.

JUNGES, A. H.; FONTANA, D. C.Avaliação do desenvolvimento das culturas de cereais de inverno no Rio Grande do Sul por meio de perfis temporais do índice de vegetação por diferença normalizada. Ciência Rural, v.39, p.1 15, 2009.

LOPEZ, C. M. L.; GERASIMOV, E.; MACHIMURA, T.; et al. Comparison of carbon and water vapor exchange of forest and grassland in permafrost regions, Central Yakutia, Russia. Agricultural and Forest Meteorology, v.148, p. 1968-1977, 2008.

MELO, R. W., FONTANA, D. C., BERLATO, M. A., DUCATI, J. R. An agrometeorological-spectral model to estimate soybean yield, applied to southern Brazil. International Journal of Remote Sensing, v.29, p.4013 - 4028, 2008.

PAULA, M. D DE; FILHO, W. P. Estimativa de Carbono em um Fragmento de Floresta Madura na Mata Atlântica Nordestina com o Uso de Índices Espectrais. In: SIMPÓSIO BRASILEIRO DE SENSORIAMENTO REMOTO, (SBSR), 2009. Natal. [Anais...], Natal: INPE, 2009. p. 2881-2888.

PONZONI, F. J; SHIMABUKURO, Y. E. Sensoriamento Remoto no Estudo da Vegetação. São José dos Campos, SP, Editora Parentese, 2007. 126p.

RIZZI, R. Geotecnologias em um sistema de estimativa da produção de soja: estudo de caso no Rio Grande do Sul. 2005. 212 p. (INPE-12271-TDI/983). Tese (Doutorado em Sensoriamento Remoto) - Instituto Nacional de Pesquisas Espaciais, São José dos Campos, 2005.

ROBERTI, D. R., MORAES, O. L. L., AMADO, T. J. C., FIORIN, J. E., JACQUES, R. J. S., TEICHRIEB C. A., ZIMERMANN, H. R., 2011. Net carbon flux in a subtropical no-tillage soybean/black oat succession in southern
Brazil. Submitted to Journal Geophysical Research Biogephysical

RODRIGUES, C.P. Estudo do Fluxo de Carbono utilizando

Geotecnologia. 2010. 92f. Dissertação (Mestrado em Sensoriamento Remoto) - Programa de Pós Graduação em Sensoriamento Remoto. Universidade Federal do Rio Grande do Sul. Porto Alegre. 2010.

ROUSE J. W.; HAAS Jr, R. H.; SCHELL J. A. and D. W. DEERING, Monitoring vegetation systems in the Great Plains with ERTS, Third ERTS Symposium, NASA SP-351 I, 309-317, 1973.

SOEGAARD, H.; JENSEN, N.O.; BOEGH, E.; HASAGER, C.B.; SCHELDE, K.; THOMSEN, A. Carbon dioxide exchange over agricultural landscape using eddy correlation and footprint modelling. Agricultural and Forest Meteorology, v.114, p. 153-173, 2003.

SULlivAN, B. W.; DORE, S.; KOLB, T. E.; HART, S. C.; MONTES-HELU, C. Evaluation of methods for estimating soil carbon dioxide efflux across a gradient of forest disturbance. Global Change Biology, v.16, p. 2449-2460, 2010 .

SUYKER, A. E., VERMA, S. B. Coupling of carbon dioxide and water vapor exchanges of irrigated and rainfed maize-soybean cropping systems and water productivity. Agricultural and Forest Meteorology, v. 150, p. 553-563, 2010.

VERMA, S.B., DOBERMANN, A., CASSMAN, K.G., WALTERS, D.T., KNOPS, J.M., ARKEBAUER, T.J., SUYKER, A.E., BURBA, G.G., AMOS, B., YANG, H., GINTING, D., HUBBARD, K.G., GITELSON, A., WATERSHEA, E.A. Annual carbon dioxide exchange in irrigated and rainfed maize-based agroecosystems. Agricultural and Forest Meteorology, v.131, p. 77-96, 2005.

WAGNER, A.P.L; WEBER.E.; FONTANA,D.C.; DUCATI,J.R.; KLERING, E. Estimativa de Área de Soja no Rio Grande do Sul Utilizando Imagens NDVI/MODIS. In: XIII Simpósio Brasileiro de Sensoriamento Remoto, 2007, Florianópolis. [Anais...] São José dos Campos: INPE, 2007. v.1. p.457-464. WATZLAWICK, L. F.; KIRCHNER, F. F.; SANQUETTA, C.R. Estimativa de biomassa e carbono em floresta com araucária utilizando imagens do Satélite Ikonos II. Ciência Florestal, Santa Maria, v. 19, n. 2, p. 169-181, 2009.

ZEEMAN, M. J.; HILLER, R.; GILGEN, A. K.; MICHNA, P.; PLÜSS, P.; BUCHMANN, N.; EUGSTER, W. Management and climate impacts on net $\mathrm{CO}_{2}$ fluxes and carbon budgets of three grasslands along an elevational gradient in Switzerland. Agricultural and Forest Meteorology, v.150, p. 519-530, 2010. 